\title{
Electron Holography for Measuring Electrostatic Potentials and Strain Fields
}

\author{
Michael Lehmann ${ }^{1}$ \\ ${ }^{1}$ Technische Universität Berlin, Institut für Optik und Atomare Physik, Berlin, Germany
}

Today's latest high-resolution transmission electron microscopes (HRTEM) with image correctors are fantastic instruments easily allowing the imaging of atomic structures with resolutions better than $100 \mathrm{pm}$ and precision of atomic column positions of a few pm. This is amongst other things the result of better control of residual aberrations and the tremendous reduction of delocalization leading to a strongly improved signal-to-noise ratio at atomic positions. However at the same time, it deteriorates the transfer of large area phase contrast represented as low spatial frequencies. Consequently, an image corrected HRTEM is a band-pass filter for phase information, good for atomic resolution, bad for measuring electrostatic potentials due to e.g. p-n junctions.

This phase problem of imaging is solved by off-axis electron holography. By means of a Möllenstedt biprism, which is only a minor supplement to a FEG-based HRTEM, amplitude and phase of the electron wave with all spatial frequencies up to the information limit are recorded within an interference experiment. Based on the fundamental considerations by Lichte [1] and Harada [2], latest holographic microscopes with at least two biprisms offer a higher experimental flexibility for adjusting width and fringe spacing of holograms [3]. In combination with improved instrumental stability, less Fresnel diffraction, reduced vignetting effect, and the acquisition of hologram series consisting of 20 or more holograms and their subsequent reconstruction considering the drifts of specimen and biprism over the series, a full reconstruction of a GaN crystal's object exit-wave up the information limit of $75 \mathrm{pm}$ of the instrument without transfer gaps has been demonstrated with a high signal-to-noise ratio allowing a precise comparison with simulations [4].

This flexible experimental setup also enables tackling fundamental questions arising from applying electron holography to real world problems: Electron holography is often used for measuring the built-in voltage of p-n junctions in doped semiconductors. However, the measured value is always smaller than the expected one as calculated from the dopant levels. It is reported that at least for Silicon providing a conductive path to ground by coating with Carbon the discrepancy is strongly reduced. Up to now, an effect only partially considered is the influence of the electron beam illumination producing electron hole pairs so that the p-n junction acts like a solar-cell illuminated in the TEM instead of light with electrons. Additionally, the high-energy electron beam leads to the generation of secondary electrons as observed as positive charging of samples, which are badly connected to ground. Using needle-shaped GaN p-n junctions prepared by FIB, careful holographic experiments (figure 1) by reducing the electron dose rate over three orders of magnitude, but acquiring hologram series with an accumulated exposure time up to $1000 \mathrm{~s}$ enabling low dose rate electron holography, have shown that indeed electron-hole pair generation plays a significant role in explaining the discrepancy whereas the generation of secondary electrons can be neglected since they do not produce a net current over the $\mathrm{p}$-n junction. Of overall importance for defined experimental conditions is a small shunt, which in the Silicon system can easily be obtained by Carbon coating, whereas in the GaN system a much larger interface resistance between bulk and conducting surface layers must exist [5].

An interesting derivative of off-axis electron holography is dark-field electron holography for measuring 
strain fields as developed by Hytch [6]. Here, a diffracted beam of an unstrained crystalline reference area is brought to interference with the corresponding reflection stemming from a strained area. The resulting phase gradient is a measure for local variations of geometrical phase. By repetition of the experiment with a second noncollinear reflection, the full 2D strain field can easily be evaluated by simple matrix algebra. Also dark-field electron holography benefits from the flexible holographic setup by strongly reducing Fresnel diffraction at the biprism filament and the recording of holographic series yielding improved signal-to-noise ratio. This is demonstrated at a GaAs mesa structure with a buried AlOx current aperture (figure 2) where the in-plane tensile strain promotes the aimed nucleation of InAs quantum dots in the middle of the aperture [7]. Quantitative comparison with corresponding calculations based on linear elasticity theory clearly shows that the strain relaxation in a TEM lamella even at a thickness of a few $100 \mathrm{~nm}$ has to be considered in the simulation. [8]

\section{References:}

[1] H. Lichte, Ultramicroscopy 64 (1996) 79.

[2] K. Harada et al., Appl. Phys. Lett. 84 (1994) 3229.

[3] F. Genz et al., Ultramicroscopy 147 (2014) 33.

[4] T. Niermann and M. Lehmann, 63 (2014) 28.

[5] Jae Bum Park et al., Appl. Phys. Lett. 105 (2014) 094102.

[6] M. Hytch et al., Nature 453 (2008) 1086.

[7] F. Kießling et al., Phys Rev. B 91 (2015) 075306.

[8] The results represent the joint efforts of the workgroup at TU Berlin especially by Tore Niermann, Felix Kießling, Jae Bum Park, and Florian Genz. The financial support through the DFG (CRC 787, TP A4) is gratefully acknowledged.

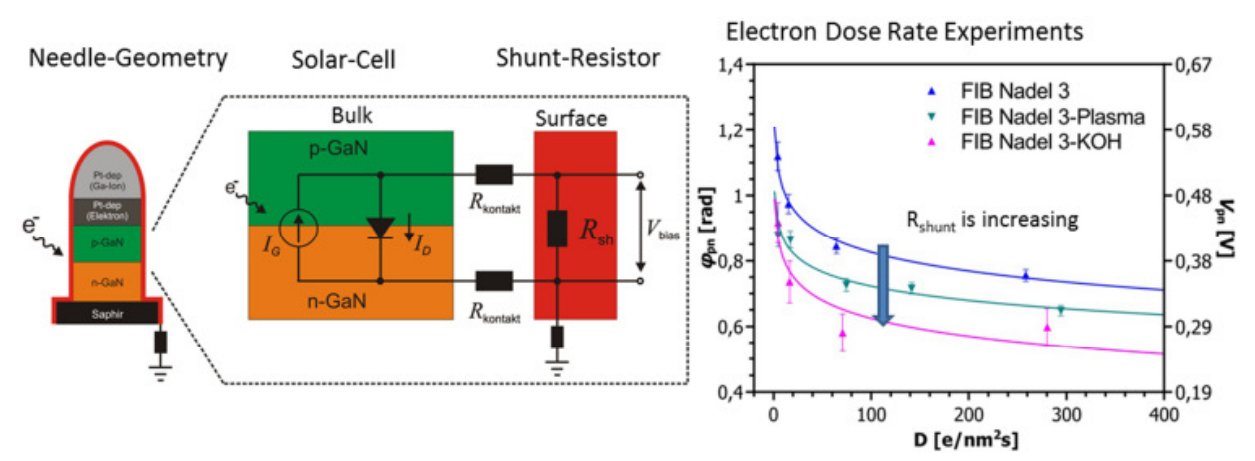

Figure 1. Equivalent circuit of the p-n junction under electron illumination. The conductive surface is altered by plasma and $\mathrm{KOH}$ etching. The measured built-in voltage is calculated for an active specimen thickness of $320 \mathrm{~nm}$.
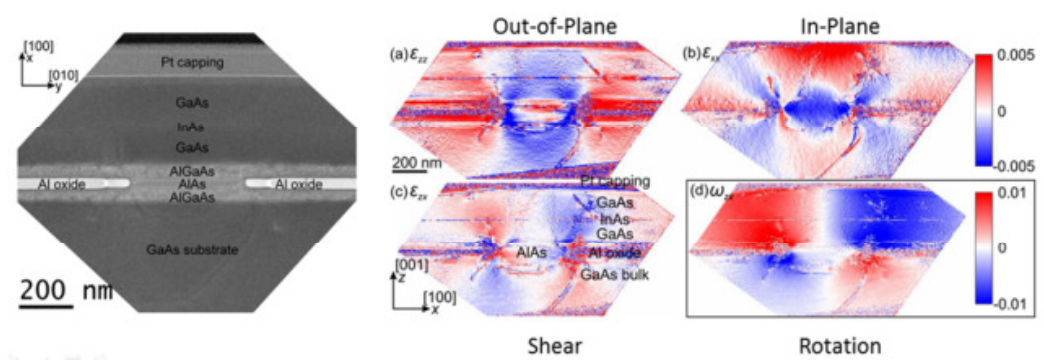

Figure 2. Left: Bright-field TEM of cross-sectional mesa structure showing clearly the extension of the AlOx current aperture. Right: Strain components as determined from dark-field electron holography. 